\title{
THE FINAL MECHANICAL DESIGN, FABRICATION, AND COMMISSIONING OF A WIRE SCANNER AND SCRAPER ASSEMBLY FOR HALO-FORMATION MEASUREMENTS IN A PROTON BEAM ${ }^{1}$
}

\author{
Robert Valdiviez, Felix Martinez, Armando Rendon, Tony Wright, John Ledford, Norm Patterson, \\ Don Bruhn, Harvey Haagenstad, J. Douglas Gilpatrick \\ Los Alamos National Laboratory \\ Derwin Martinez, General Atomics \\ Jim O’Hara, Honeywell
}

\begin{abstract}
The $6.7 \mathrm{MeV}, 100 \mathrm{~mA}$ proton beam being produced in the Low Energy Demonstration Accelerator (LEDA) RFQ is being injected into a 52 magnet lattice in order to study the charged-beam phenomenon known as beam halo [1]. Quadrupole magnets in the lattice are purposely mismatched to cause or amplify halo formation in the beam. Interceptive diagnostics that consist of a thin wire and a paddle type device called a scraper are placed in the beam to obtain charge-distribution data. The charge-distribution data is used to create a current-density distribution plot of the beam at the probed location [2]. This paper describes the mechanical design, fabrication, and commissioning of the interceptive diagnostic devices and the assembly that carries them.
\end{abstract}

\section{INTRODUCTION}

A photograph of one of the 18 measuring assemblies, or axis manufactured is shown in Figure 1. The inner assembly that resides under vacuum when installed on the beam line consists of an outer and inner frame structure. The outer frame structure is fixed to the vacuum housing when installed, and provides support for the inner-movable frame that carries the wire and two scrapers. The wire traverses the entire beam cross-section, while the scrapers are inserted only to a depth of approximately $2 \mathrm{rms}$ beam widths, or about $2 \mathrm{~mm}$ away from the beam's center. Signal wires are connected to the wire and scraper devices, and pass through the bellows and into a signal box on the outer portion of the whole assembly. Isolation electrical connectors in the signal box allow the transition from vacuum to air, and pass the signals on to cables that run to the diagnostic electronics that are located away from the experiment lattice. A stepper motor and encoder combination is used to control and monitor the motion of the inner frame structure.

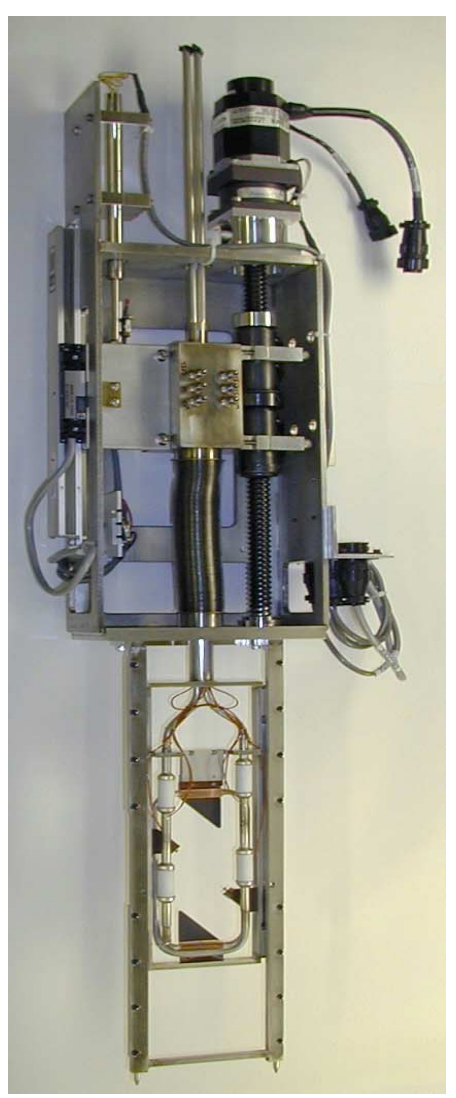

Figure 1: Halo Scraper/Wire Scanner Assembly

\section{THE WIRE SCANNER}

\subsection{The Sensing Wire}

The thin sensing wire is an important and integral part of this diagnostic assembly. Data from the 33 micron diameter carbon wire is used to obtain a current-density distribution plot of the beam. The current-density distribution plot provides a cross-sectional profile of the beam, and allows for determining how far the scrapers can be inserted into the beam.

\footnotetext{
${ }^{1}$ This work sponsored by the U.S. Department of Energy
} 
The peak heat flux that the wire is exposed to is $1400 \mathrm{~kW} / \mathrm{cm}^{2}$. The peak heat flux occurs for the beam parameters of a $1-\mathrm{mm}$ rms size, $100-\mathrm{mA}$ current, a 20-microsecond pulse length, and a beam repetition rate of $1 \mathrm{~Hz}$. The predicted pseudo-steady-state peak temperature for the wire under these conditions is $1717 \mathrm{~K}$ $\left(1444{ }^{\circ} \mathrm{C}\right)$. The limiting temperature is $1800 \mathrm{~K}\left(1527^{\circ} \mathrm{C}\right)$ where the onset of thermionic emission is believed to occur. Actual testing of the wire early on in the experimenting verified that the thermionic emission temperature is indeed near $1800 \mathrm{~K}$. Figure 2 is a plot of the predicted wire temperature where the beam on and off pulsing effect can be seen by the temperature rising and falling in step with the beam repetition rate.

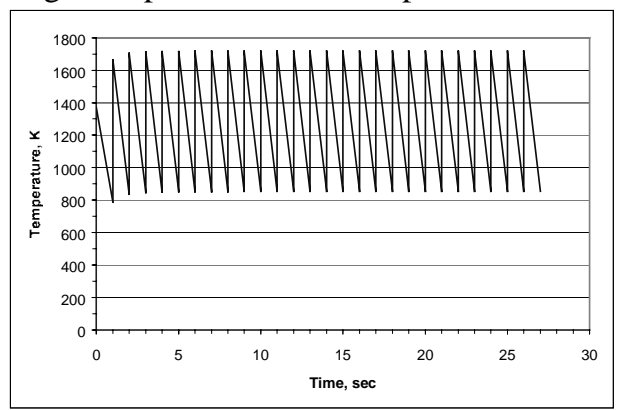

Figure 2: Predicted Wire Temperatures

\subsection{Mounting of the Sensing Wire}

A key part of the assembly is the mounting of the 33 micron wire onto the inner movable frame. A small spring-loaded subassembly was designed to grasp the wire and hold it in place for making wire scan measurements. The requirements for the holding subassembly are: i) grip and hold the wire with no slippage, ii) maintain electrical conductivity in the wire circuit, iii) prevent the wire from sagging due to thermal expansion, and iv) electrically isolate the wire from the support frame. A spring loaded clamp was designed to grasp the wire. Figure 3 is an illustration of the clamp mechanism. The clamp consists of a tapered two-jawed collet, a matching tapered collar, a compression spring, a retaining ring, and a dielectric housing. The dielectric housing is made of Vespel [3]. The Vespel holder is bolted to the inner movable frame. The Vespel holder provides both mechanical support and electrical isolation. Two mounting clamp subassemblies are used on each measuring axis to hold one wire in place. A signal wire of the wire scanner assembly is soldered directly to the collar of the clamp assembly in order to carry the wire scan signal to the detection electronics.

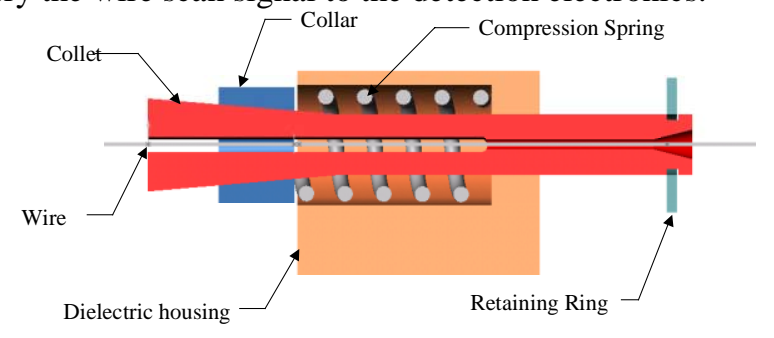

Figure 3: Spring-Loaded Clamp
The wire is mounted into two clamps that are fastened to the inner movable frame of the scanner assembly by first threading the wire into the collet with the spring load relaxed. The collar is slid into place on the collet in order to grip the wire. The collet-wire-collar combination is pressed lightly against the spring, and the wire is threaded into the opposite facing clamp assembly. This spring compression will determine the preload that is placed on the wire. The wire is fixed in the second clamp by repeating the same process as was used on the first clamp. The end of the wire protruding from the back end of the each clamp is trimmed off. The wire is now properly mounted in the assembly.

\section{THE BEAM SCRAPER}

\subsection{The Scraper Analysis and Design}

The peak target heat flux that the scraper is intended to be exposed to is $610 \mathrm{~kW} / \mathrm{cm}^{2}$. The peak predicted temperature for the scraper is $493{ }^{\circ} \mathrm{C}$. The peak predicted stress is $31 \mathrm{MPa}$. The beam interaction region of the scraper oscillates between minimum values and these peaks as the beam is pulsed on and off. This creates a fatigue loading situation. The scraper design has been tested in a high heat flux application, and was found to be able to maintain its integrity in excess of 180,000 cycles [4]. This number of cycles allows for ample halo testing in the LEDA lattice. Figure 4 illustrates the scraper design.

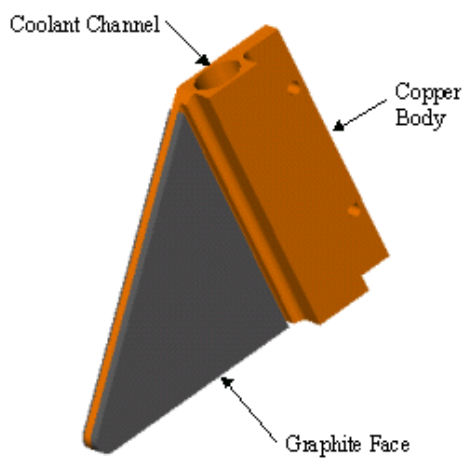

Figure 4: Beam Halo Scraper

\subsection{The Scraper Fabrication}

A scraper is fabricated by first machining the copper body and graphite face plates. The copper body is brazed to the stainless steel cooling tubes in the first braze of the process. Next, the graphite face plates are brazed onto the scrapers. Although only one face intercepts the proton beam two graphite face pieces are brazed on to the scraper. Two graphite pieces are brazed on to balance the bending loads placed on the scraper during the brazing cool-down cycle and prevent permanent warping of the scraper. Final machining is done on the scraper to create sharp edges on the beam intercepting portion, and remove excess graphite. 


\section{ASSEMBLY FABRICATION}

A diagnostic assembly, or axis, is assembled by taking a completed scraper subassembly that consists of 2 scrapers connected with coolant tubes and electrical isolators, and mounting it to an inner frame structure. The electrical isolators are placed in the coolant tubing to allow the use of a guard voltage that limits the water channel resistivity effects on the scraper detected signal [5]. The scraper subassembly coolant tubes are seal welded to the tubes that are already attached to the outer portion of the axis. An outer frame structure is assembled around the inner frame structure and bolted to the vacuum sealing flange of the axis. This outer frame structure provides support for the moving inner frame that carries the scrapers and sensing wire. The electrical signal wires that are already connected to the vacuum feed-through box mounted on the outer portion of the axis are soldered to the scraper and wire circuits. Bias and guard voltage wires are also soldered into place. The 33 micron diameter sensing wire is installed into its holders. Final hand cleaning is done on the vacuum exposed surfaces of the lower assembly to complement the previous individual part cleaning done for vacuum service. The axis is now ready for positional measurement, bake-out, and installation.

\section{POSITIONAL MEASUREMENTS OF THE SENSING COMPONENTS}

The physical distances from the scraper edges to the sensing wire are important parameters to quantify for each diagnostic assembly. These dimensions are used in the control logic to determine the distance from each scraper's edge to the beam's center. The beam center and rms width are calculated from the wire scan data. This data is then used to set the limiting insertion position for each scraper [6].

Each $\mathrm{X}$ and $\mathrm{Y}$ axis is placed in a measuring fixture in order to physically measure the wire to scraper edge separation distances. This fixture is a vacuum box enclosure identical to the units installed on the beam line, with the exception that this measuring box has additional fiducials added to enable the measuring. An axis is inserted into the measuring box and fastened into place just as it would be on the beam line. Two clear optical targets are placed in front of and behind the box, and are aligned to the bore of the box to create a centerline that is orthogonal to the travel of the axis. A telescope is positioned to view along this invisible centerline by aligning it to the optical targets. The telescope is positioned away from the measuring box to enable a depth of field that focuses the targets simultaneously. The object being measured then appears in focus, and in between the two optical targets in the bore of the box. The telescope enables viewing the object and the proper zero mark alignment. The wire is aligned to the targets on a zero mark, the encoder set point is zeroed, and the axis is moved to align a scraper edge with the same zero mark. The encoder reading is recorded as the separation distance from the wire to the scraper edge. The same process is repeated for the other scraper on the same axis.

\section{PREPARATION OF AN AXIS FOR INSTALLATION}

Each axis undergoes a bake-out procedure after being measured, and prior to being installed on the beam line. Special canisters were fabricated to accommodate the baking process. This baking process allows for the beam-line target vacuum level of $1.0 \mathrm{E}-7$ Torr to be reached in a relatively short amount of time. The bake out is done at a temperature of $180{ }^{\circ} \mathrm{C}$ for eight hours. After an axis cools from the baking process the enclosing canister is back-filled with dry nitrogen gas. The axis remains under this gas blanket until it is removed for installation on the beam line. Room-air exposure time for an axis is short, on the order of two hours.

\section{BEAM COMMISSIONING OF THE ASSEMBLY}

All eighteen measuring axes are operational on the beam line. The greatest beam-exposure heat load for a wire has been estimated to be $1400 \mathrm{~kW} / \mathrm{cm}^{2}$. The greatest heat flux exposure for a scraper has been estimated at $600 \mathrm{~kW} / \mathrm{cm}^{2}$. The maximum beam pulse measurement cycles that an axis has experienced is approximately 4,600 cycles. This data indicates that the predicted performance of the diagnostic assembly should be realized throughout actual use. Merged wire scan and scraper data indicate the formation of beam halo for mismatched conditions in the lattice, as would be expected. This beam data has been collected without any damage to the diagnostic's sensing surfaces that intercept the beam.

\section{REFERENCES}

[1]T. Wangler, "Beam Halo in Proton Linac Beams", LINAC 2000, Monterey, CA, August 2000.

[2] Gilpatrick, J. D., 2000, "Beam Diagnostic Instrumentation for the Low-Energy Demonstration Accelerator (LEDA): Commissioning and Operational Experience", Proceedings, European Particle Accelerator Conference 2000, Vienna, Austria.

[3] Vespel is a registered trademark of the DuPont Company, DuPont Engineering Polymers, 2000.

[4] R. Valdiviez, "The High-Heat Flux Testing of an Interceptive Device for an Intense Proton Beam", these proceedings.

[5] M. Gruchalla, "Beam Profile Wire-Scanner/HaloScraper Sensor Analog Interface Electronics", these proceedings.

[6]J. Kamperschroer , "Analysis of Data From the LEDA Wire Scanner/Halo Scraper", these proceedings. 